\title{
White dwarf spins from low-mass stellar evolution models
}

\author{
M. P. L. Suijs ${ }^{1}$, N. Langer ${ }^{1}$, A.-J. Poelarends ${ }^{1}$, S.-C. Yoon $^{2}$, A. Heger ${ }^{2,3}$, and F. Herwig ${ }^{4}$ \\ 1 Astronomical Institute, Utrecht University, PO Box 80000, 3508 TA, Utrecht, The Netherlands \\ e-mail: n.langer@astro.uu.nl \\ 2 Department of Astronomy and Astrophysics, University of California, Santa Cruz, CA 95060, USA \\ 3 Theoretical Astrophysics Group, T-6, MS B227, Los Alamos, NM 87545, USA \\ ${ }^{4}$ Keele Astrophysics Group, School of Physical and Geographical Sciences, Keele University, Staffordshire ST5 5BG, UK
}

Received 17 January 2008 / Accepted 15 February 2008

\begin{abstract}
Context. The prediction of the spins of the compact remnants is a fundamental goal of the theory of stellar evolution. Aims. Here, we confront the predictions for white dwarf spins from evolutionary models, including rotation with observational constraints.

Methods. We perform stellar evolution calculations for stars in the mass range $1 \ldots 3 M_{\odot}$, including the physics of rotation, from the zero age main sequence into the TP-AGB stage. We calculate two sets of model sequences, with and without inclusion of magnetic fields. From the final computed models of each sequence, we deduce the angular momenta and rotational velocities of the emerging white dwarfs.

Results. While models including magnetic torques predict white dwarf rotational velocities between 2 and $10 \mathrm{~km} \mathrm{~s}^{-1}$, those from the nonmagnetic sequences are found to be one to two orders of magnitude larger, well above empirical upper limits.

Conclusions. We find the situation analogous to that in the neutron star progenitor mass range, and conclude that magnetic torques may be required to understand the slow rotation of compact stellar remnants in general.
\end{abstract}

Key words. stars: rotation - stars: evolution - stars: magnetic fields - stars: white dwarfs

\section{Introduction}

During the last decade, rotation and rotationally-induced transport processes have been a major focus of the theory of massive star evolution (Maeder \& Meynet 2000; Heger et al. 2000). Evolutionary models, without magnetic field-induced internal angular momentum transport, predict spins of newly-born neutron stars, which are $1 . . .2$ orders of magnitude above those deduced from the youngest Galactic pulsars (Ott et al. 2006). However, the magnetic torques proposed by Spruit $(1998,2002)$ provide enough coupling between the rapidly-rotating core and the slowly-rotating envelope in post-main sequence stars to produce the right amount of core spin-down (Heger et al. 2005; Petrovic et al. 2005).

In models of low mass stars, the role of nonmagnetic rotational transport processes for the determination of the white dwarf spin is rather negligible, which leads to rapidly-rotating CO-cores (Langer et al. 1999; Palacios et al. 2003, 2006, cf. below). The huge shear above these rotating $\mathrm{CO}$-core during the thermally-pulsing AGB phase inhibits the s-process by swamping the ${ }^{13} \mathrm{C}$-pocket with the neutron poison ${ }^{14} \mathrm{~N}$ (Herwig et al. 2003; Siess et al. 2004). Analogous to the situation in massive stars, it appears that such rapidly spinning cores in low-mass star models also contradict direct observations of compact stellar remnants. In a recent analysis of the $\mathrm{Ca}$ line profiles of a large sample of DA white dwarfs, Berger et al. (2005) concluded that their rotational velocities are generally below $10 \mathrm{~km} \mathrm{~s}^{-1}$, which is the smallest upper limit derived from spectroscopy so far. They conclude that the predicted white dwarf spin from the rotating, nonmagnetic evolutionary models of Langer et al. (1999) can be ruled out. This conclusion is confirmed by Kawaler (2004), who argues that the rotation rate of ZZ Ceti pulsators is even at least one order of magnitude slower than Berger et al.'s upper limit.

Internal magnetic torques have been suggested as an agent to spin down the cores of white dwarf progenitors during the giant stage (Spruit 1998). In this paper, we investigate whether magnetic torques, as computed in Spruit (2002), are able to alleviate the problem of slowly-spinning white dwarfs. Eggenberger et al. (2005) already used the same physics to compute the angular momentum evolution of a solar mass star, with the result that the flat internal angular velocity profile of our Sun could be recovered.

Magnetic torques may not be the only mechanism to provide a spin-down of the stellar core. For example, Charbonnel \& Talon (2005) invoked angular momentum transport though gravity waves (Zahn et al. 1997) to explain the slow rotation of the Solar core. However, here we concentrate on the magnetic torque mechanism, as it gives promising results for massive stars and can be readily applied to the low-mass regime.

\section{Method}

We use a 1D hydrodynamic stellar evolution code (Yoon et al. 2006, and references therein). It includes diffusive mixing due to convection, semiconvection (Langer et al. 1985), and thermohaline mixing as in Wellstein et al. (2001). This code includes the effect of the centrifugal force on the stellar structure, and time-dependent chemical mixing and transport of angular momentum due to rotationally-induced instabilities (Heger et al. 2000). We also include chemical mixing and transport of angular momentum due to magnetic fields (Spruit 2002), as in 
Table 1. Initial mass $M_{\mathrm{i}}$, initial equatorial velocity $v_{\mathrm{rot}, \mathrm{i}}$, adopted white dwarf mass $M_{\mathrm{WD}}$ and radius $R_{\mathrm{WD}}$ final core angular momentum $J_{\mathrm{c}, \mathrm{f}}$, final mass averaged specific core angular momentum $j_{\mathrm{c}, \mathrm{f}}$ and expected equatorial rotation velocity $\left(v_{\mathrm{rot}, \mathrm{WD}}\right)$ from Eq. (2), for our magnetic (upper 4 lines) and nonmagnetic models.

\begin{tabular}{ccccccc}
\hline \hline$M_{\mathrm{i}}$ & $\begin{array}{c}v_{\mathrm{rot}, \mathrm{i}} \\
M_{\odot}\end{array}$ & $\begin{array}{c}M_{\mathrm{WD}} \\
\mathrm{km} \mathrm{s}^{-1}\end{array}$ & $\begin{array}{c}R_{\mathrm{WD}} \\
10^{-2} R_{\odot}\end{array}$ & $\begin{array}{c}J_{\mathrm{c}, \mathrm{f}} / 10^{46} \\
\mathrm{~g} \mathrm{~cm}^{2} \mathrm{~s}^{-1}\end{array}$ & $\begin{array}{c}j_{\mathrm{c}, \mathrm{f}} / 10^{13} \\
\mathrm{~cm}^{2} \mathrm{~s}^{-1}\end{array}$ & $\begin{array}{c}v_{\text {rot, } \mathrm{WD}} \\
\mathrm{km} \mathrm{s}^{-1}\end{array}$ \\
\hline 1.0 & 2 & 0.550 & 1.25 & 5.00 & 4.54 & 2.6 \\
1.5 & 45 & 0.575 & 1.20 & 5.83 & 5.07 & 3.0 \\
2.0 & 140 & 0.600 & 1.19 & 8.05 & 6.71 & 4.5 \\
3.0 & 250 & 0.680 & 1.08 & 15.4 & 11.3 & 9.1 \\
1.0 & 2 & 0.550 & 1.25 & 8.07 & 7.33 & 4.2 \\
1.5 & 45 & 0.575 & 1.20 & 110 & 95.6 & 56 \\
2.0 & 140 & 0.600 & 1.19 & 329 & 274 & 180 \\
3.0 & 250 & 0.680 & 1.08 & 458 & 336 & 220 \\
\hline
\end{tabular}

Heger et al. (2005) and Petrovic et al. (2005). We assume rigid rotation in convection zones.

We calculate the evolution of solar metallicity stars that start rigidly rotating at the zero-age main sequence, for initial masses of 1.0 $M_{\odot}, 1.5 M_{\odot}, 2.0 M_{\odot}$, and 3.0 $M_{\odot}$, with and without the effects of magnetic torques. We chose the initial equatorial velocities of these models to be 2, 45, 140, and $250 \mathrm{~km} \mathrm{~s}^{-1}$ (Tassoul 2000). For the solar mass models, we assume that the angular momentum loss due to magnetic braking connected with the solar-type wind is already over once we start our calculations (cf. Eggenberger et al. 2005). All but our $3 M_{\odot}$ models evolve through the core helium flash. Throughout the evolution of all models, we used the mass-loss rate of Reimers (1975). The choice of the AGB mass-loss rates does not affect our results significantly. We stopped the calculations after at least five and at most 28 thermal pulses, beyond which the CO-core angular momentum is not expected to change significantly (cf., Sect. 3).

We computed the surface rotational velocities of the white dwarfs emerging from our model sequences as follows. First, we fixed the final white dwarf mass according to the initialfinal mass relation from Weidemann (2000). These white dwarf masses (cf. Table 1) are generally slightly larger than the final CO-core masses of our models. However, as we shall see below, the total angular momentum of the CO-core does not change any more during its growth on the TP-AGB, and therefore, even though we corrected our final CO-core masses in the mentioned way, our final angular momenta do not need to be adjusted.

The surface rotational velocities are then calculated assuming rigid rotation inside the white dwarf. This is well justified due to the short timescale of angular momentum redistribution $\left(\sim 10^{8} \mathrm{yr}\right)$ inside even the nonmagnetic models (Yoon \& Langer 2004). We then use the gyration radii, $H$, for polytropes derived by Motz (1952). For a white dwarf with a polytropic index $n=1.5$, mass $M_{\mathrm{WD}}$, radius $R_{\mathrm{WD}}$ (we employ the white dwarf mass-radius relation of Hamada \& Salpeter 1961), and angular momentum $J_{\text {tot }}$, it is (Table 1 in Motz 1952):

$$
\left(\frac{H}{R_{\mathrm{WD}}}\right)^{2}=0.20502 \text {. }
$$

With $\omega=J / I$ and $I_{\mathrm{WD}}=H^{2} M_{\mathrm{WD}}$ we obtain the surface velocity

$v_{\mathrm{rot}, \mathrm{WD}}=\omega_{\mathrm{WD}} R_{\mathrm{WD}}=\frac{J_{\mathrm{tot}}}{I_{\mathrm{WD}}} R_{\mathrm{WD}}=\frac{J_{\mathrm{tot}}}{0.20502 R_{\mathrm{WD}} M_{\mathrm{WD}}}$,

where $\omega_{\text {WD }}$ is the angular velocity of the white dwarf. We have tested this procedure by computing rotating cold white dwarf

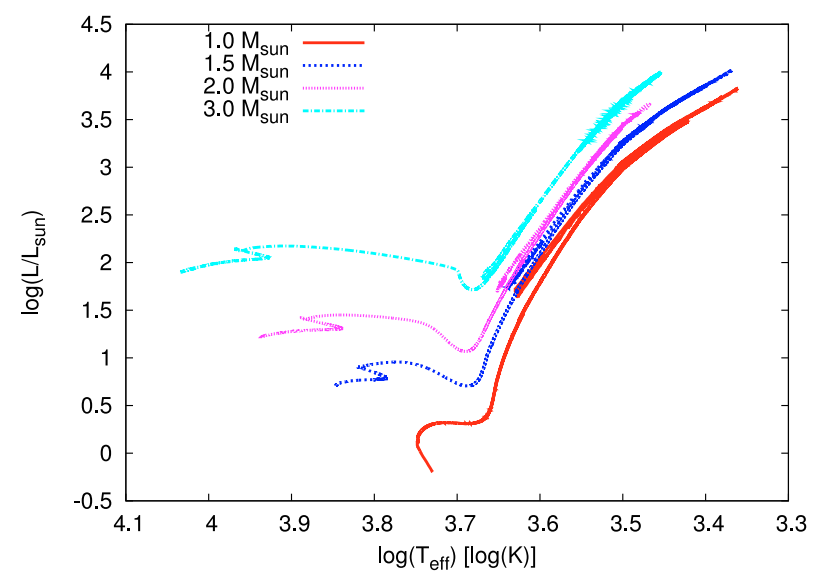

Fig. 1. Evolutionary tracks of the four sequences which include magnetic torques, in the Hertzsprung-Russell diagram (cf. Table 1).

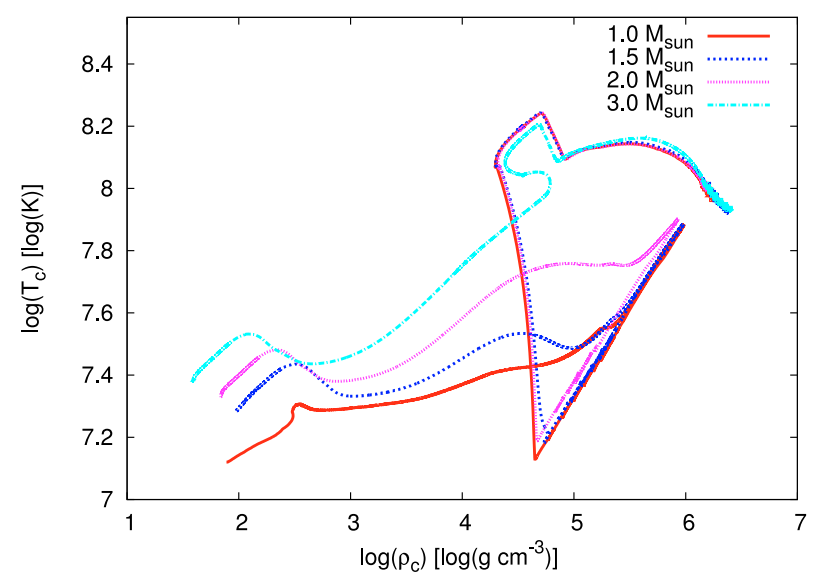

Fig. 2. Evolution of the four sequences which include magnetic torques, in the central density $\left(\rho_{\mathrm{c}}\right)$ versus central temperature $\left(T_{\mathrm{c}}\right)$ plane.

models with our stellar evolution code and find it accurate to within $10 \%$ for the slowly-rotating cases considered here.

\section{Results}

The evolutionary tracks of our four magnetic models in the HR-diagram are shown in Fig. 1, while Fig. 2 displays their evolution in the $T_{\mathrm{c}}-\rho_{\mathrm{c}}$-plane. The tracks of our nonmagnetic sequences would be indistinguishable on these plots. Figure 2 shows that during the late AGB evolution, the CO-cores of our models are degenerate and cooling. Their central densities are close to that of cold white dwarfs in the considered mass range, i.e., $\rho_{\mathrm{c}} \simeq 3 \ldots 6 \times 10^{6} \mathrm{~g} \mathrm{~cm}^{-3}$ for $M_{\mathrm{WD}}=0.55 M_{\odot} \ldots 0.7 M_{\odot}$.

In order to visualize the angular momentum distribution in our models, we show diagrams using the integrated angular momentum $J\left(M_{\mathrm{r}}\right)=\int_{0}^{M_{\mathrm{r}}} j\left(M_{\mathrm{r}}\right) \mathrm{d} M_{\mathrm{r}}$ divided by $M_{\mathrm{r}}^{5 / 3}$, as a function of the mass coordinate $M_{\mathrm{r}}$. While a homogeneous, rigidlyrotating body results in a horizontal line in such a diagram, the $J\left(M_{\mathrm{r}}\right)$-profiles from different evolutionary stages trace the flow of angular momentum through the mass shells, since $J\left(M_{\mathrm{r}}\right)$ and $J\left(M_{\mathrm{r}}\right) M_{\mathrm{r}}^{-5 / 3}$ remain constant in a given mass shell if no angular momentum is transported through this shell.

Figure 3 displays $J$-profiles from six evolutionary stages for our $1.5 M_{\odot}$ model, both with and without the effects of magnetic torques. For the nonmagnetic model, the deepest extension of the convective envelope is down to about $0.2 M_{\odot}$. Therefore, the 


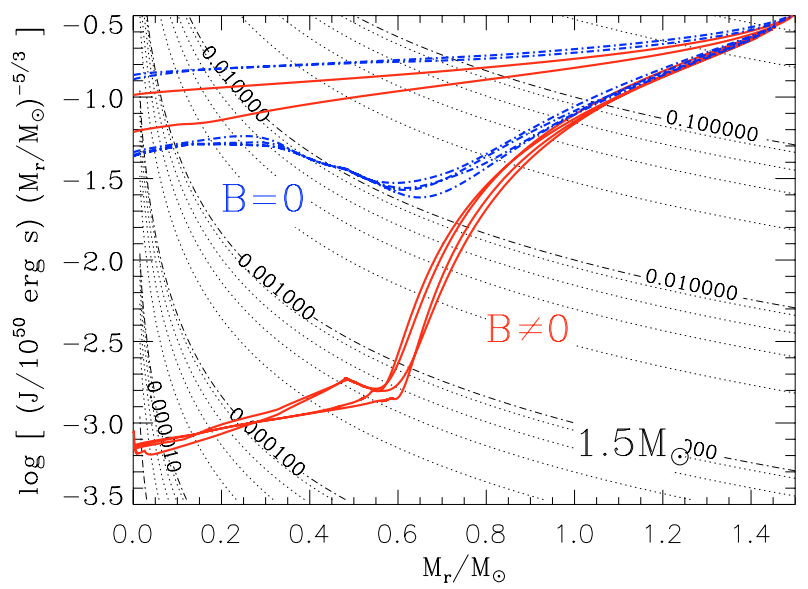

Fig. 3. Integrated angular momentum $J\left(M_{\mathrm{r}}\right)=\int_{0}^{M_{\mathrm{r}}} j(m) \mathrm{d} m$ divided by $M_{\mathrm{r}}^{5 / 3}$ as a function of the mass coordinate, at different times for the magnetic (full-drawn red lines) and nonmagnetic (dashed blue lines) $1.5 M_{\odot}$ sequences. The contours display levels of constant $J$, labeled with $\log \left(J / 10^{50} \mathrm{erg} \mathrm{s}\right)$. The values for the dotted contours are 3, 5, 7, and 9 times the value of the labeled contour below them. From high to low, the six lines per sequence represent the following evolutionary stages: start main sequence $\left(X_{\mathrm{c}}=0.7\right)$, main sequence $\left(X_{\mathrm{c}} \sim 0.25\right)$, start core He burning $\left(Y_{\mathrm{c}} \sim 0.95\right)$, He burning $\left(Y_{\mathrm{c}} \sim 0.4\right)$, start TP phase and during TP phase ( $>5$ pulses).

helium core is built-up starting with the initial angular momentum of the inner $0.2 M_{\odot}$, i.e., about $10^{48} \mathrm{~cm}^{2} \mathrm{~s}^{-1}$. During the growth of the helium core on the RGB, material from the former bottom of the convective envelope is incorporated, which is very slowly rotating and which, in its inner parts, has a specific angular momentum several orders of magnitude below that of the material in the helium core. Thus, effectively, the helium core grows in mass without gaining angular momentum. This feature is continued for the $\mathrm{CO}$-core during the AGB-evolution. As a consequence, the $J$-profiles in Fig. 3 follow the line of $J=10^{48} \mathrm{~cm}^{2} \mathrm{~s}^{-1}$ in the mass range $0.2 M_{\odot}<M_{\mathrm{r}}<0.6 M_{\odot}$ during the post-main sequence evolution. Other processes than convection are clearly negligible in this case. The specific angular momentum in the inner $0.6 M_{\odot}$ core of this model has been lowered by about a factor 6 , from the ZAMS to the white dwarf stage. For the other nonmagnetic models, this factor is rather similar.

For the magnetic $1.5 M_{\odot}$ sequence, Fig. 3 shows a drop of core angular momentum already during core hydrogen burning. This is a consequence of the magnetic fields enforcing close-torigid rotation. However, the main drain of core angular momentum occurs between core hydrogen exhaustion and core helium ignition, i.e., during the RGB phase. The total loss of core angular momentum here is about a factor of 20 larger than in the nonmagnetic case, i.e., a factor of 120 in total. Figure 4, which compares the final angular momentum distributions of our magnetic and nonmagnetic models, shows that the situation is similar for all the studied cases, except for the solar mass models, where the initial angular momentum was already rather low.

\section{Discussion}

Figure 5 summarizes our results by showing the initial and the final average, specific core angular momentum of our model. It also contains the corresponding result from Heger et al. (2005) for a $15 M_{\odot}$ star. Observational constrains from white dwarf spectroscopy, pulsation analyses of ZZ Ceti stars, magnetic

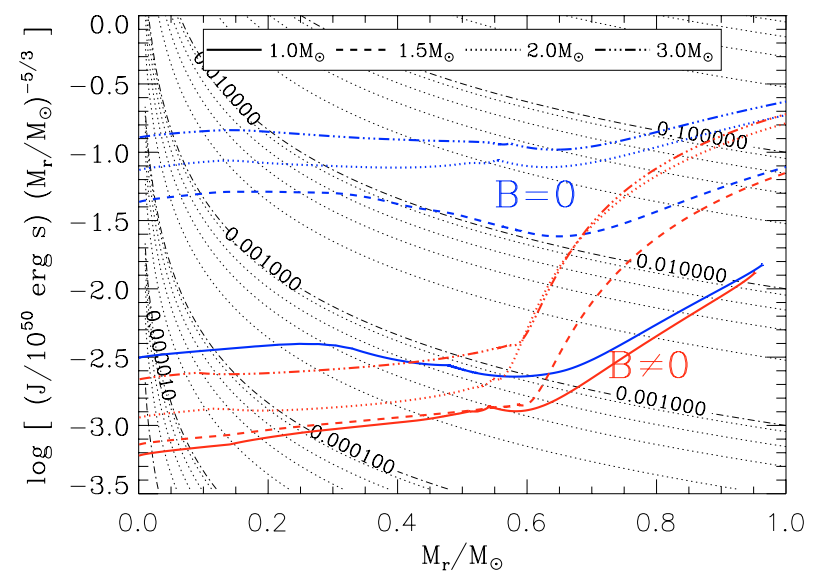

Fig. 4. Integrated angular momentum $J\left(M_{\mathrm{r}}\right)=\int_{0}^{M_{\mathrm{r}}} j(m) \mathrm{d} m$ divided by $M_{\mathrm{r}}^{5 / 3}$, as a function of the mass coordinate for the final stellar models of our sequences (cf. Table 1). The background contours display levels of constant $J$, as in Fig. 3.

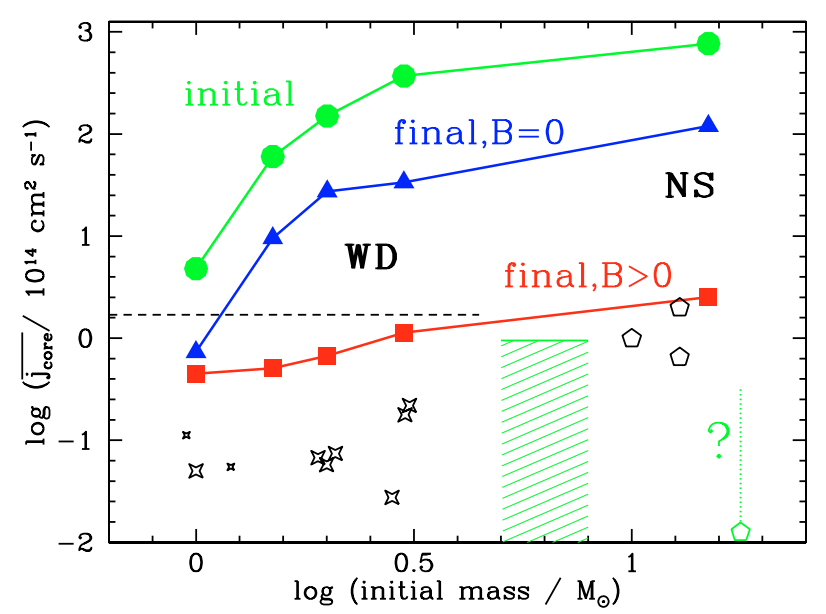

Fig. 5. Average core specific angular momentum of our initial and final models (cf. Table 1 and Fig. 4) versus initial stellar mass (full drawn lines). Points for $15 M_{\odot}$ stars computed with the same physics from Heger et al. (2005) have been added. The upper line corresponds to the initial models. Filled triangles corresponds to the final models of the nonmagnetic sequences, and filled squares to the final models of the magnetic sequences. The dashed horizontal line indicates the spectroscopic upper limit on the white dwarf spins obtained by Berger et al. (2005). Star symbols represent astroseismic measurements from ZZ Ceti stars (Bradley 1998, 2001; Dolez 2006; Handler 2001; Handler et al. 2002; Kepler et al. 1995; Kleinmann et al. 1998; Winget et al. 1994), where smaller symbols correspond to less certain measurements. The green hatched area is populated by magnetic white dwarfs (Ferrario \& Wickramasinghe 2005; Brinkworth et al. 2007). The three black open pentagons correspond to the youngest Galactic neutron stars (Heger et al. 2005), while the green pentagon is thought to roughly correspond to magnetars (Camilo et al. 2007), where the vertical-dotted green line indicates the possibility that magnetars are born with higher core angular momentum.

white dwarfs, and young neutron stars are also plotted on this figure.

In the initial mass range considered here, as well as in the massive star regime, it is clear that the nonmagnetic models fail by a large margin to comply with the observations. Only for solar mass stars, where magnetic braking due to the solartype wind leads to a low initial angular momentum, could the nonmagnetic model be marginally consistent with the empirical 
data. However, our assumption was that the slow surface rotation of these stars early during hydrogen burning has slowed down the rotation of the whole stellar interior. Eggenberger et al. (2005) showed that exactly this might have been performed by the B-fields produced due to the Spruit (2002) dynamo.

The magnetic low-mass models all fall below the spectroscopic limit on white dwarf rotation of Berger et al. (2005). Toward more massive stars, the agreement with observations seems good. The neutron star spins are well recovered, and the range of spins of the magnetic white dwarfs (which are presumed to have more massive progenitors than typical white dwarfs; Ferrario \& Wickramasinghe 2005) reaches up to the line connecting $3 M_{\odot}$ and $15 M_{\odot}$. The partly, much-slower rotation of the magnetic white dwarfs could be explained by additional magnetic braking due to stellar winds in the white dwarf progenitors. However, our magnetic models still predict white dwarf rotation rates in the $1 M_{\odot}$ to $3 M_{\odot}$ range which are about one order of magnitude larger than what is found in pulsating white dwarfs (ZZ Ceti stars). This implies that the white dwarfs should lose additional angular momentum, either after they are born, or still inside their progenitor stars.

In the first case, one may speculate whether the situation in analogous to the neutron star case: for pulsars it is well known that they spin down with time. As the ZZ Ceti stars have a cooling age of about $10^{9} \mathrm{yr}$, they might have lost angular momentum on their cooling track. However, estimating the spin-down time through a magnetic wind by $\tau_{1}=\dot{M}^{-\frac{1}{2}}(M / B R)(2 G M / R)^{\frac{1}{4}}$ (Justham et al. 2006) would allow for a significant effect at most in strongly-magnetic white dwarfs, and spin-down through electromagnetic radiation, with $\tau_{2}=M c^{3} /\left(B^{2} \Omega^{2} R^{4}\right)$ is always insignificant. In the above expressions, $M, R, \Omega$ and $B$ are white dwarf mass, radius, surface magnetic field strength and spin frequency.

In the second case, perhaps an additional internal angular momentum transport mechanism in white dwarf progenitors is required. While transport through gravity waves has been suggested as such (Zahn et al. 1997), we point out that for stars above $\sim 1.3 M_{\odot}$, the core spin-down is likely to occur during the post-main sequence evolution since on the main sequence these stars are still rapid rotators. It is unclear at present whether gravity waves can transport angular momentum through the shell source in the giant stage. On the other hand, the magnetic angular momentum transport has been shown by Zahn et al. (2007) to be more complex than in the picture of Spruit (2002). Also, Yoon et al. (2008) find strong poloidal fields may be generated by convective cores, and suggest that their influence on angular momentum transport may be comparable to that of the fields suggested by Spruit.

\section{Conclusions}

While nature may be more complex, it is worthwhile to attempt to understand the spins of compact stellar remnants with a single theoretical approach for all initial masses. We showed above that angular momentum transport through rotationally-induced magnetic fields, according to Spruit (2002), provides a major improvement of the predicted spins of white dwarfs and neutron stars. Magnetic angular momentum transport also appears the most promising candidate to bridge the still existing gap between observed and predicted white dwarf spins at low mass (see Fig. 5).

If internal magnetic torques in their progenitors are indeed resopnsible for the slow rotation of compact stars in the Milky Way, then there may be little room for an important role of angular momentum in their formation process, at least in single stars. This may have implications for understanding the progenitors and the formation mechanism of magnetars (Sawai et al. 2008) and long gamma-ray bursts (Woosley \& Bloom 2006).

Acknowledgements. A.H. performed this work under the auspices of the National Nuclear Security Administration of the US Department of Energy at Los Alamos National Laboratory under Contract No. DE-AC52-06NA25396, and was supported by the DOE Program for Scientific Discovery through Advanced Computing (SciDAC; DE-FC02-01ER41176).

\section{References}

Berger, L., Koester, D., Napiwotzki, R., Reid, I. N., \& Zuckerman, B. 2005, A\&A, 444, 565

Bradley, P. A. 1998, ApJS 116, 307

Bradley, P. A. 2001, ApJ 552, 326

Brinkworth, C. S., Burleigh, M. R., \& Marsh, T. R. 2007, in 15th European

Workshop on White Dwarfs, ed. R. Napiwotzki, \& M. R. Burleigh, San Francisco, ASP Conf. Ser., 372, 183

Camilo, F., Ransom, S. M., Halpern, J. P., \& Reynolds, J. 2007, ApJ, 666, L93

Charbonnel, C., \& Talon, S. 2005, Science, 309, 2189

Dolez, N., Vauclair, G., Kleinman, S. J., et al. 2006, A\&A, 446, 237

Eggenberger, P., Meynet, G., \& Maeder, A. 2005, A\&A, 440, L9

Ferrario, L., \& Wickramasinghe, D. T. 2005, MNRAS, 356, 615

Hamada, T., \& Salpeter, E. E. 1961, ApJ, 134, 683

Handler, G. 2001, MNRAS, 323, L43

Handler, G., Romero-Colmenero, E., \& Montgomery, M. H. 2002, MNRAS, 335,399

Heger, A., Langer, N., \& Woosley, S. E. 2000, A\&A, 2000, ApJ, 528, 368

Heger, A., Woosley, S. E., \& Spruit, H. C. 2005, ApJ, 626, 350

Justham, S., Rappaport, S., \& Podsiadlowski, P. 2006, MNRAS, 366, 1415

Kawaler, S. D. 2004, in Stellar Rotation, ed. A. Maeder, \& P. Eenens, ASP, IAU Symp., 215, 561

Herwig, F., Langer, N., \& Lugaro, M. 2003, ApJ, 593, 1056

Kepler, S. O., Giovannini, O., Wood, M. A., et al. 1995, ApJ, 447, 874 Kleinman, S. J., Nather, R. E., Winget, D. E., et al. 1998, ApJ, 495, 424 Langer, N., El Eid, M. F., \& Fricke, K. J. 1985, A\&A, 145, 179

Langer, N., Heger, A., Wellstein, S., \& Woosley, S. E. 1999, A\&A, 346, L37

Maeder, A., \& Meynet, G. 2000, ARA\&A, 38, 143

Maeder, A., \& Meynet, G. 2005, A\&A, 440, 1041

Motz, L. 1952, ApJ, 115, 562

Ott, C. D., Burrows, A., Thompson, T. A., Livne, E., \& Walder, R. 2006, ApJS, 164,130

Palacios, A., Talon, S., Charbonnel, C., \& Forestini, M. 2003, A\&A, 399, 603

Palacios, A., Charbonnel, C., Talon, S., \& Siess, L. 2006, A\&A, 453, 261

Petrovic, J., Langer, N., Yoon, S.-C., \& Heger, A. 2005, A\&A, 435, 247

Reimers, D. 1975, Mem. Soc. Liege, 8, 369

Sawai, H., Kotake, K., \& Yamada, S. 2008, ApJ, 672, 465

Siess, L., Goriely, S., \& Langer, N. 2004, A\&A, 415, 1089

Spruit, H. C. 1998, A\&A, 333, 603

Spruit, H. C. 2002, A\&A, 381, 923

Tassoul, J. L. 2000, Stellar Rotation (Cambridge University Press)

Weidemann, V. 2000, A\&A, 363, 647

Wellstein, S., Langer, N., \& Braun, H. 2001, A\&A, 369, 939

Winget, D. E., Nather, R. E., \& Clemens, J. C. 1994, ApJ, 430, 839

Woosley, S. E., \& Bloom, J. S. 2006, ARA\&A, 44, 507

Yoon, S.-C., \& Langer, N. 2004, A\&A, 419, 623

Yoon, S.-C., Langer, N., \& Norman, C. 2006, A\&A, 460, 199

Yoon, S.-C., et al. 2008, Proc. IAU Symp., 250, in press

Zahn, J.-P., Talon, S., \& Matias, J. 1997, A\&A, 322, 320

Zahn, J.-P., Brun, A. S., \& Mathis, S. 2007, A\&A, 474, 145 